\title{
Artesunate Improves Drug Resistance of Lung Carcinomas Via Reg- ulation of MiR-493-5p
}

\author{
Hong-rui Zhang, Yan Zhang, Fu-lian Qu, Pei-jie Liu, Zhi-qiao Xu* \\ Department of tumor center, Kaifeng central hospital, Kaifeng, 475000, China.
}

\section{Abstract}

Objective: The aim of this study was to explore the potential involvement of the miR-493-5p/BRCA1 pathway in the anti-cell drug resistance activity of artesunate in lung carcinomas.

Method: Two drug-resistant lung carcinoma cell lines, A549 and SBC-3, resistant to cisplatin (DDP) and adriamycin (ADM), respectively, and their parental cell lines (A549 and SBC-3) were used in this study. Cell viability was determined by an MTT assay. The relative expression of miR-493-5p and BRCA1 mRNA was analyzed by quantitative real-time PCR. The protein expression of BRCA1, breast cancer resistance protein (BCRP), and (p)PI3K/AKT was determined by a Western blot.

Result: Artesunate attenuated the viability of A549/DDP and SBC-3/ADM cells and suppressed the expression of BCRP, an important multidrug resistance protein in lung carcinoma cells, accompanied by upregulation of miR-493-5p and downregulation of BRCA1. In artesunate-treated A549/DDP and SBC-3/ADM cells, miR493-5p silencing reversed the anti-cell drug resistance activity of artesunate and resulted in increased cell viability and BRCP expression. Simultaneously, miR-493-5p silencing contributed to BRCA1 upregulation and phosphorylation of PI3K and AKT. As shown by a luciferase reporter gene assay, BRCA1 was a target gene for miR-493-5p, and its expression was negatively regulated by miR-493-5p. miR-493-5p silencing reversed the anticell drug resistance activity of artesunate, and this finding was further supported by a murine tumorbearing model.

Conclusion: The data support the anti-cell drug resistance activity of artesunate in lung carcinoma cells and sheds light on the role of the miR-493-5p/BRCA1 pathway in this process.

Keywords: Artesunate; BRCA1; drug resistance, miR-493-5p; lung carcinoma

\section{INTRODUCTION}

Lung carcinomas are a leading cause of cancer-related deaths worldwide ${ }^{[1]}$. According to data from the National Cancer Center of China, among all types of cancer in China, lung cancer is responsible for the highest morbidity and mortality. The treatment of lung carcinomas is hampered by the ineffectiveness of current therapy, leading to a poor prognosis ${ }^{[2]}$. Multidrug resistance of lung carcinoma cells remains a major problem that has yet to be resolved ${ }^{[2]}$.

Antitumor agents for cancers have increasingly attracted attention, particularly studies of Chinese traditional plant compounds. One such agent is artesunate, which has antineoplasmic activity. Artesunate belongs to drug monomers and is derived from the leaves of Artemisia annua L (also known as qinghao or sweet wormwood), a plant used in traditional Chinese medicine ${ }^{[3]}$ and re-

*Corresponding author: Zhi-qiao Xu

Mailing address: Department of tumor center, Kaifeng central hospital, Hejie road no.85, Kaifeng, Henan, 475000, China.

E-mail: esc7312@163.com

Received: 20 April 2018 Accepted: 26 June 2018 nowned globally for its antimalaria activity ${ }^{[4]}$. Recently, artesunate has been recognized as an anticancer agent in various cancers, such as breast cancer ${ }^{[5]}$, cervical cancer ${ }^{[6]}$, and lung cancer ${ }^{[7]}$. In addition, accumulating evidence points to anti-tumor cell drug resistance activity of artesunate in prostate cancer ${ }^{[8]}$, head and neck cancer ${ }^{[9]}$, and multiple myelomas ${ }^{[10]}$. However, to date, there is no direct evidence of an effective role of artesunate in multidrug resistance of lung carcinoma. MicroRNAs (miRNAs) are a class of small noncoding RNAs with a length of $\sim 22$ nucleotides. Although they have no protein-coding ability, miRNAs are attracting widespread attention because of their regulatory role in functional protein expression via post-transcription modification processing. Unexpectedly, in previous studies, miRNAs interplaying with critical proteins developed into a molecular mechanism for drug resistance of lung carcinomas ${ }^{[11-13]}$. miR-493 has been recently recognized as an antitumor miRNA in breast cancer ${ }^{[14]}$, gastric cancer ${ }^{[15]}$, colon cancer ${ }^{[16]}$, and lung cancer ${ }^{[17]}$. Research also showed that miR-493 was involved in regulating chemoresistance of gastric cancer cells ${ }^{[18]}$. 

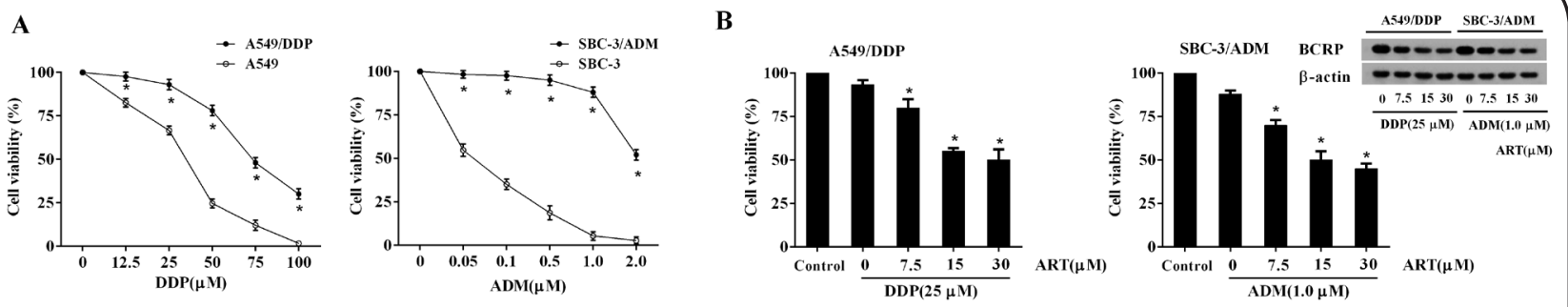

Figure 1. Effect of artesunate on drug resistance of lung carcinoma cells. (A) An MTT assay detected the cell viability of lung carcinoma cell lines (A549 and SBC-3) and paired drug-resistant lung carcinoma cell lines (A549/DDP and SBC-3/ADM) after treatment with different doses of DDP or ADM. (B) Cell viability analysis of A549/DDP and SBC-3/ADM cells with combined treatment of DDP or ADM and doses of artesunate. (C) A Western blot assay determined BCRP expression in A549/DDP and SBC-3/ ADM cells with combined treatment of DDP or ADM and doses of artesunate. ${ }^{*} \mathrm{P}<0.05$ compared with A549, SBC-3, or A549/DDP with $25 \mu \mathrm{mol} / \mathrm{L}$ DDP treatment or SBC-3/ADM with $1.0 \mu \mathrm{mol} / \mathrm{L}$ ADM treatment.

We previously investigated the role of artesunate in drug resistance of lung carcinoma cells and explored the potential involvement and downstream pathway of miR-493 in this process. Drug resistant-associated proteins regulated by miRNAs underlie the regulatory mechanism of drug resistance of cancer in various cancers ${ }^{[11]}$. The aim of this study was to determine the possibly regulated BRCA1, a tumor suppressor gene and drug-resistance related gene, in lung carcinomas ${ }^{[19]}$, by miR-493 in artesunate-treated lung carcinoma cells.

\section{MATERIALS AND METHODS}

\section{Cell culture and treatment}

Two drug-resistant lung carcinoma cell lines, A549/ DDP (resistant to cisplatin) and SBC-3/ADM (resistant to adriamycin), and their parental cell lines, A549 and SBC-3 (obtained from ATCC) were cultured in RPMI1640 medium, supplemented with $10 \%$ fetal calf serum and 1\% penicillin-streptomycin solution. A549/ DDP and SBC-3/ADM were established by A549 and SBC-3 cells step by step and treated with increasing concentrations of DDP or ADM. The cells were maintained in a humidified atmosphere at $37^{\circ} \mathrm{C}$ with $5 \%$ $\mathrm{CO}_{2}$, and the medium was replaced with new medium every 2-3 days.

To evaluate the cell cytotoxic activity of DDP or ADM, the A549/DDP and A549 cells were exposed to DPP at concentrations of $12.5,25,50,75$, and $100 \mu \mathrm{M}$, and the SBC-3/ADM and SBC-3 cells were exposed to ADM at concentrations of $0.05,0.1,0.5,1.0$, and $2.0 \mu \mathrm{M}$. The cells were treated with combination treatment $(25 \mu \mathrm{M}$ DPP and 1.0 $\mu \mathrm{M} \mathrm{ADM)} \mathrm{and} \mathrm{different} \mathrm{doses} \mathrm{of} \mathrm{artesu-}$ nate $(7.5,15$, and $30 \mu \mathrm{M}$ ) or $15 \mu \mathrm{M}$ artesunate (A549/ DDP and SBC-3/ADM cells).

\section{MTT assay}

An MTT assay was performed to evaluate the cytotoxic activity of DDP, ADM, and artesunate. The cells $(1 \times$
$10^{4} / \mathrm{ml}$ ) were seeded into a 96 -well plate, and different doses of DPP, ADM, or artesunate were added. The cells were cultured for $48 \mathrm{~h}$ and added with an MTT reaction kit. After incubation for $4 \mathrm{~h}$, the reaction in each well was stopped. The generated formazan crystals of samples were resolved by wells to which $150 \mu \mathrm{l} /$ of dimethyl sulfoxide (DMSO) was added per well. The OD value of each dissolution mixture was examined at $570 \mathrm{~nm}$ by a microplate reader.

Western blot analysis of the protein expression of breast cancer resistance protein (BCRP), BRCA1, PI3K/ AKT, and (p)PI3K/AKT

Cells or tumor tissues were collected for total protein extraction with RIPA lysis buffer, supplemented with protease inhibitor. Protein samples were quantified by the bicinchoninic acid method. Subsequently, an equal quantity of protein from all the protein samples was subjected to SDS-polyacrylamide gel electrophoresis. The proteins in the gel were then transferred to polyvinylidene fluoride membrane (Millipore), followed by incubation with primary antibodies against BCRP, BRCA1, PI3K/AKT, and (p)PI3K/AKT at $4^{\circ} \mathrm{C}$ overnight. The next day, the membranes were washed and incubated with horseradish peroxidase-conjugated secondary antibody for $2 \mathrm{~h}$ at room temperature. Finally, target genes were visualized using an ECL Western blotting substrate kit. The electrophoretic band of $\beta$-actin was regarded as an internal control.

Quantitative real-time PCR (qRT-PCR) determination of the relative expression of miR-493-5p and BRCA1 mRNA

Cells or tumor tissues were collected for total RNA isolation using an RNA extraction kit (Tiangen, Beijing, China), according to the manufacturer's protocol. RNAs were subjected to reverse transcription using an miRNA First Strand Synthesis Kit (Takara, Dalian, China) or PrimeScript $^{\mathrm{TM}}$ RT Master Mix (Takara, Dalian, China). 
A

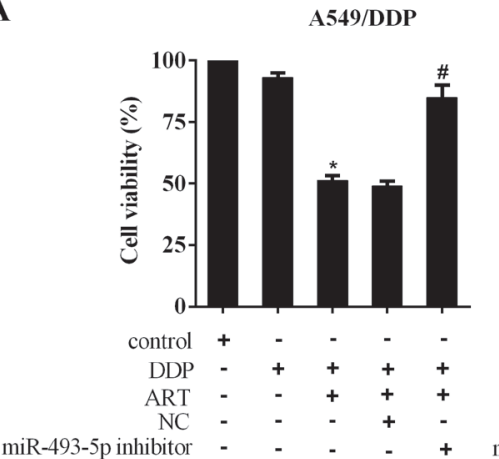

SBC-3/ADM

B

Figure 3. miR-493-5p silencing attenuated the anticell viability effect of artesunate. (A) Cell viability analysis of A549/DDP and SBC-3/ADM cells with co-treatment of $25 \mu \mathrm{mol} / \mathrm{L}$ DDP or $1.0 \mu \mathrm{mol} / \mathrm{L}$ ADM, $15 \mu \mathrm{mol} / \mathrm{L}$ ART, and an miR-493-5p inhibitor. (B) Determination of the BCRP level in A549/DDP and SBC-3/ADM cells co-treated with $25 \mu \mathrm{mol} / \mathrm{L}$ DDP or $1.0 \mu \mathrm{mol} / \mathrm{L}$ ADM or $15 \mu \mathrm{mol} /$ L ART and an miR-493-5p inhibitor. NC, negative control. ${ }^{\mathrm{P}}<0.05$ compared with DDP or ADM; \#P $<0.05$ compared with DDP + ART + NC or $\mathrm{ADM}+\mathrm{ART}+\mathrm{NC}$.

For the real-time PCR reaction, the cDNA product was amplified by SYBR ${ }^{\circledR}$ Premix Ex Taq ${ }^{\mathrm{TM}}$ II (Takara, Dalian, China) using an Applied Biosystems 7300 Fast Real-Time PCR System. The U6 gene or GAPDH was used as an endogenous control.

\section{Luciferase reporter gene assay}

Basing on the predicted pairing bases between the BRCA1 3'-untranslated region (3'-UTR) and miR-493$5 \mathrm{p}$, the BRCA1 $3^{\prime}$-UTR was amplified and packaged into a luciferase vector forming a BRCA1 3'-UTR-WT (wild type) recombinant. A fragment of mutant pairing bases of BRCA1 3'-UTR was constructed into a BRCA1 3'-UTRMut (mutant type) recombinant and regarded as a pos- itive control. To determine the regulatory role of miR493-5p in BRCA1 3'-UTR activity, a BRCA1 3'-UTR-WT recombinant or BRCA1 3 '-UTR-Mut recombinant was co-transfected with an miR-439-5p inhibitor or miR439-5p mimic into A549 cells or A549/DDP cells. After $48 \mathrm{~h}$ transfection, the cells were harvested for luciferase activity analysis.

\section{Murine tumor-bearing model}

BALB/c nude mice (6 weeks) were subcutaneously injected with $2 \times 10^{6}$ A549/DPP cells and randomly divided into four groups according to drug administration (DDP, DDP + artesunate, DDP + artesunate + negative control (NC), DDP + artesunate + lentivirus (LV) miR-

A
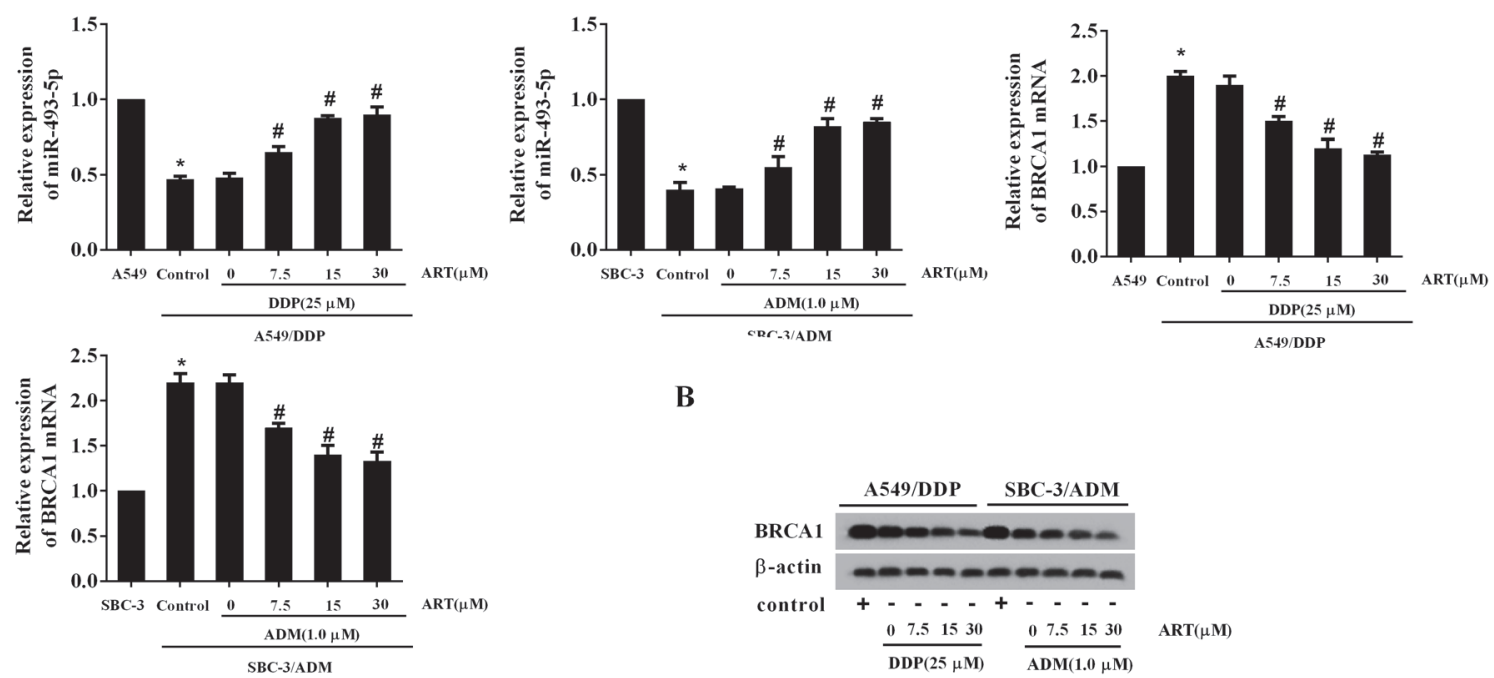

B

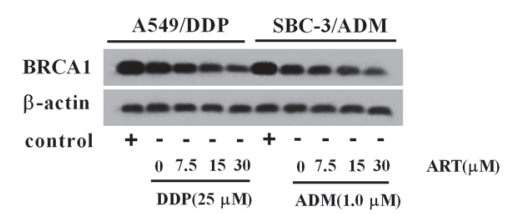

Figure 2. Effect of artesunate on the expression of miR-493-5p and BRCA1. (A) qRT-PCR was performed to detect the relative expression of miR-493-5p and BRCA1 mRNA in A549/DDP and SBC-3/ADM cells with combined treatment of DDP or ADM and doses of artesunate. (B) Determination of BRCA1 protein level in A549/DDP and SBC-3/ADM cells with combined treatment of DDP or ADM and doses of artesunate. ${ }^{*} \mathrm{P}<0.05$ compared with A549 or SBC-3; ${ }^{\#} \mathrm{P}<0.05$ compared with A549/DDP with $25 \mu$ mol/L DDP treatment or SBC-3/ADM with $1.0 \mu \mathrm{mol} / \mathrm{L}$ ADM treatment. 
493-5p inhibitor). After the cell injection, tumor volume was measured every $4 \mathrm{~d}$. Drug administration commenced when the tumor volume was $100 \mathrm{~mm}^{3}$. DDP was delivered via an intraperitoneal injection, and artesunate was delivered via the oral route. A lentivirus constructed miR-493-5p inhibitor (LV-miR-493-5p inhibitor) was administrated via an intravenous injection through the tail. Tumor volume was measured continuously for $4 \mathrm{wk}$. The animals were then sacrificed, and tumor tissues were removed for determination of miR-493-5p and BRCA1 expression.

\section{Statistical analysis}

Statistical analysis was performed with GraphPad Prism 5 software and version 15.0 SPSS software. Data were analyzed by a one-way analysis of variance or the student's t test and represented as mean \pm SD. A twotailed $P$ value less than 0.05 was considered statistically significant.

\section{RESULTS}

Artesunate improved the drug resistance of lung carcinoma cells

First, we evaluated the degree of drug resistance of two drug-resistant lung carcinoma cells lines, A549/DDP and SBC-3/ADM. The results revealed that A549/DDP and SBC-3/ADM showed resistance to DDP and ADM and that they exhibited higher cell viability than paired nondrug-resistant cells (A549 and SBC-3) at different drug doses (Figure 1A). The drug resistance of A549/ DDP to DDP and SBC-3/ADM to ADM was attenuated by artesunate in a dose-dependent manner (Figure 1B). In addition, the protein level of BCRP, an important multidrug resistance protein in lung carcinoma cells [20] decreased in different doses of artesunate-treated A549/DDP and SBC-3/ADM cells, pointing to the anti-cell drug resistance activity of artesunate in lung carcinoma cells.

Artesunate contributed to upregulation of miR-493-5p and downregulation of BRCA1 in A549/DDP and SBC3/AD cells

The potential involvement of miR-493-5p and BRCA1 in the anti-cell drug resistance activity of artesunate was determined by examination of their expression levels in artesunate-treated A549/DDP and SBC-3/ADM cells. As shown in Figure 2A, the relative expression level of miR-493-5p gradually increased and that of BRCA1 mRNA decreased in accordance with an increase in the dose of artesunate. Similar to BRCA1 mRNA, the expression level of the BRCA1 protein in A549/DDP and SBC-3/ADM cells was also reduced, depending on the artesunate dose administered (Figure 2B).

miR-493-5p silencing reversed the anti-cell drug resistance activity of artesunate

To confirm the regulatory role of miR-493-5p in the anti-cell drug resistance activity of artesunate, we explored the effect of miR-493-5p silencing on the viability of A549/DDP and SBC-3/ADM cells and found that miR-493-5p silencing reversed the anti-cell drug resistance activity of artesunate and restored cell viability (Figure 3A). Furthermore, BCRP expression was upregulated in miR-493-5p inhibitor-transfected A549/DDP and SBC-3/ADM cells (Figure 3B).

miR-493-5p silencing was negatively correlated with the upregulation of BRCA1 pathway

Next, we investigated the potentiating effect of miR493-5p on the BRCA1 pathway in artesunate-treated A549/DDP and SBC-3/ADM cells. miR-493-5p silencing resulted in upregulation of BRCA1 mRNA and the BRCA1 protein (Figs $4 \mathrm{~A}$ and $\mathrm{B}$ ). As the PI3K/AKT path-
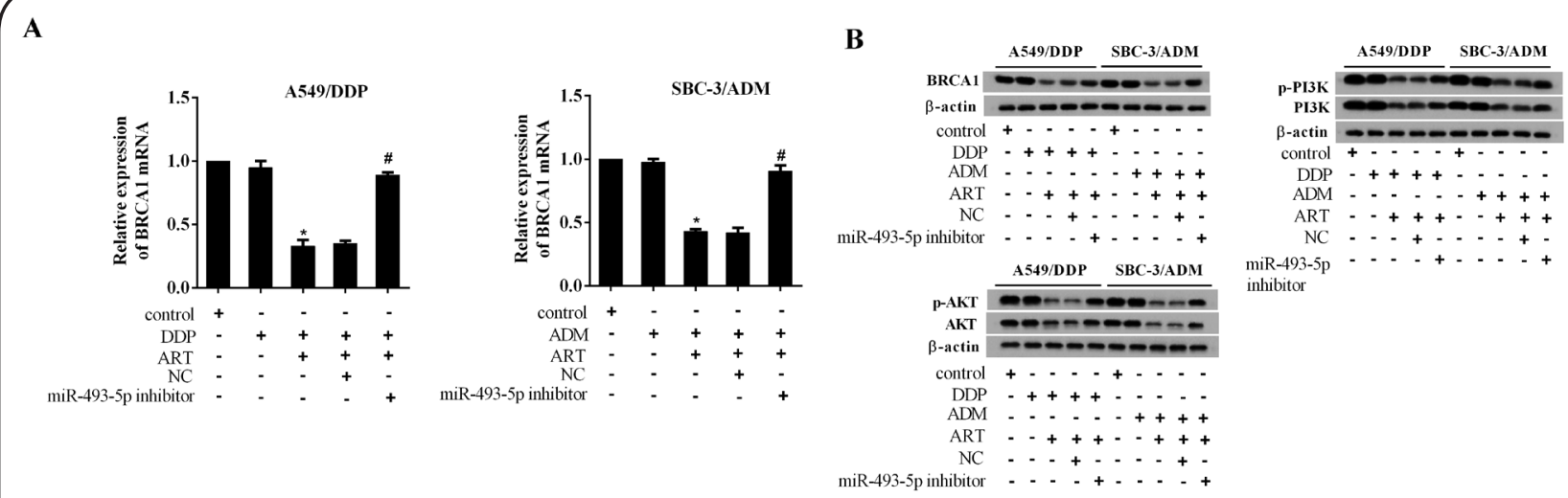

Figure 4. Effect of miR-493-5p silencing on BRCA1 and the PI3K/AKT pathway in artesunate-treated A549/DDP and SBC-3/ ADM cells. The cells were treated as in Figure 3 and harvested for analysis of (A) relative mRNA expression of BRCA1 and (B) protein expression of BRCA1, PI3K, p-PI3K, AKT, and p-AKT. *P < 0.05 compared with DDP or ADM; \#P < 0.05 compared with DDP + ART + NC or $\mathrm{ADM}+\mathrm{ART}+\mathrm{NC}$. 
A

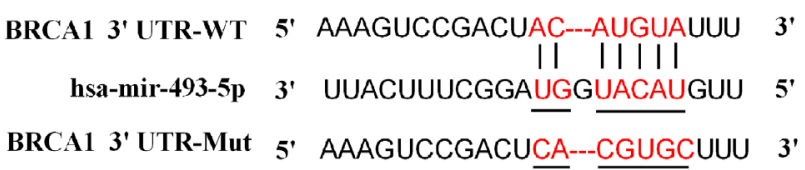

C
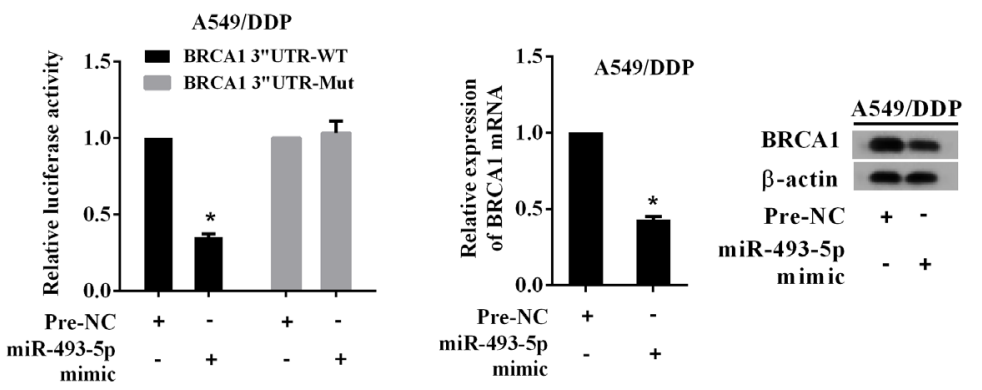

B
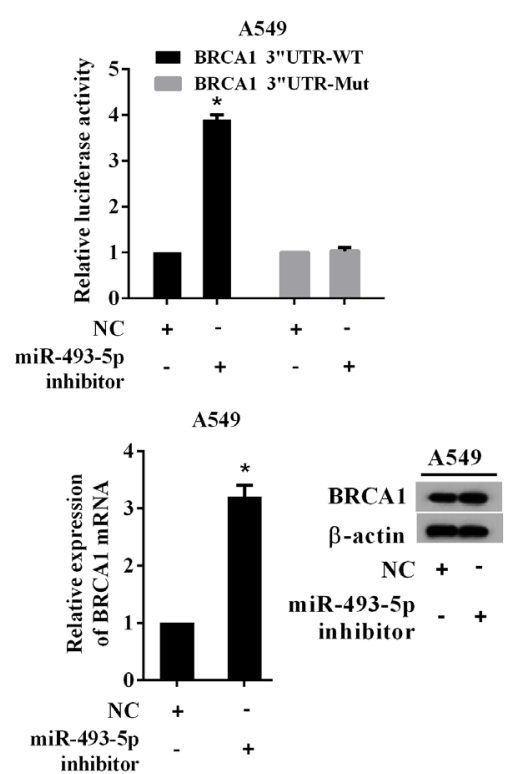

Figure 5. miR-493-5p targetedBRCA1. (A) Representation of base pairing between miR-493-5p and BRCA1 3'-UTR-WT and matched base pairing between miR-493-5p and BRCA1 $3^{\prime}$-UTR-Mut. (B) A luciferase reporter assay was performed to analyze relative luciferase activity of the 3 '-UTR of BRCA1 in A549 cells and A549/DDP cells. (C) Determination of the BRCA1 mRNA and BRCA1 protein in A549 cells after transfection with an miR-493-5p inhibitor and in A549/DDP cells after transfection with an miR493-5p mimic. $* \mathrm{P}<0.05$ compared with NC or pre-NC.

way is a downstream pathway for BRCA1 in drug resistance of NSCLC cell lines ${ }^{[20]}$, we determined the phosphorylated level of PI3K and AKT in artesunate-A549/ DDP and SBC-3/ADM cells. The results revealed that artesunate inhibited the phosphorylation of PI3K and AKT. Notably, the inhibitory effect of artesunate on PI3K and AKT phosphorylation was alleviated by miR493-5p silencing, suggesting the responding BRCA1/ PI3K/AKT pathway to miR-493-5p in anti-cell drug resistance activity of artesunate (Figure 4B).

\section{miR-493-5p directly targeted BRCA1}

Figure 5A shows the base pairing between miR-493-5p and BRCA1 3'-UTR, indicating potential direct binding between them. The results of the luciferase reporter gene assay demonstrated the regulatory role of miR493-5p in BRCA1 3'-UTR activity in A549 and A549/ DDP cells (Figure 5B). Consequently, BRCA1 mRNA and BRCA1 protein expression were enhanced by miR-493$5 p$ silencing in A549 cells and inhibited by miR-493-5p overexpression (Figure 5C).

Determination of the effect of miR-493-5p silencing on tumor growth in tumor-bearing mice following artesunate administration

The effect of miR-493-5p silencing on the anti-cell drug resistance activity of artesunate was studied in an A549/DDP xenograft mice model by observing tumor growth (Figure 6A). Compared with DDP, artesunate treatment suppressed tumor volume, and the inhibitory effect of artesunate on tumor growth was substantially weakened in an LV-miR-493-5p inhibitor tumor xenograft model. In accordance with the results of the cell experiments, the analysis of miR-493-5p and BRCA1 in tumor xenografts showed that miR-493-5p was upregulated in mice with artesunate administration and downregulated in LV-miR-493-5p inhibitor tumor xenografts. The BRCA1 protein level was lower in artesunate-treated tumors and higher when miR-493-5p was silenced (Figure 6B).

\section{DISCUSSION}

ADM- and DDP-based chemotherapies are commonly used clinically for pulmonary malignant tumors. However, resistance of cancer cells to these agents results in treatment failure ${ }^{[21]}$. Recently, Chinese traditional medicines have attracted attention as effective antitumor agents for various cancers. The antitumor activity of artesunate has been demonstrated in a wide range of cancers ${ }^{[22]}$. The present study showed that artesunate treatment resulted in an effective improvement in the cell drug resistance of lung carcinoma to ADM and DDP. Thus, artesunate can be regarded as an effective anti-cell drug resistance agent.

Evidences pointing to the tight relationship between miRNAs and carcinogenesis promote the investigation of mechanism by which miRNAs mediate multi-drug resistance of cancer cells ${ }^{[23]}$. The data in the present 
A

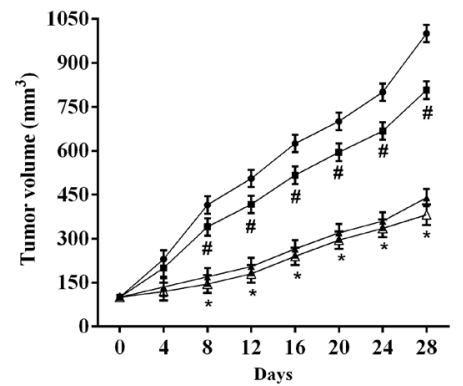

$\rightarrow$ DDP

$\triangle \mathrm{DDP}+\mathrm{ART}$

- DDP+ART+NC

DDP+ART+miR-493-5p
inhibitor
B

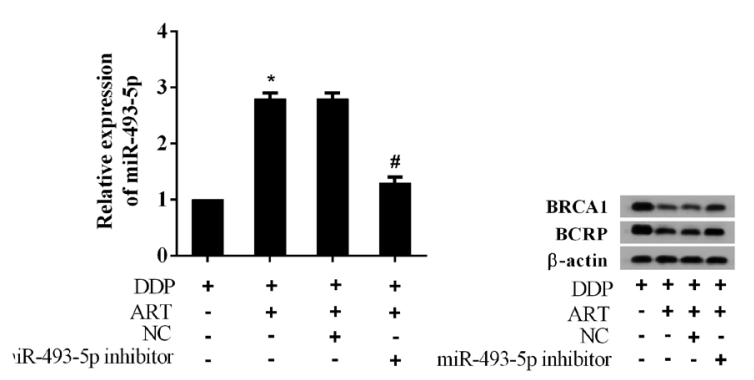

Figure 6. miR-493-5p silencing promoted tumor growth in an artesunate-treated A549/DDP xenograft murine model. (A) Tumor volume was measured on day 4 after DDP injection and artesunate administration every $4 \mathrm{~d}$. (B) Determination of miR-493-5p and BRCA1 in an A549/DDP xenograft. *P < 0.05 compared with DDP; \#P < 0.05 compared with DDP + ART + NC.

study suggested that miR-493, a recently identified antitumor miRNA ${ }^{[24,25]}$, mediated the process of anti-cell drug resistance of lung carcinoma cells by artesunate. Previous research reported that miR-493 was downregulated in several cancers. Accordingly, upregulation of miR-493 by vector-mediated gene transfection contributed to inhibition of proliferation and metastasis of gastric cancer cells ${ }^{[26]}$ and impairment of cancer cell growth and migration of lung cancer ${ }^{[17]}$ and bladder cancer ${ }^{[25]}$. Our data suggested that miR-493-5p was increasingly expressed in the process of anti-cell drug resistance of lung carcinoma cells by increased concentrations of artesunate in both A549/DDP and SBC-3/ ADM cells, pointing to the involvement of miR-493 in the anti-cell drug resistance activity of artesunate. This idea was confirmed by observation of the reversal effect of miR-493-5p silencing on cell viability protection of artesunate. These data confirmed the anti-chemoresistant role of miR-493 ${ }^{[15]}$.

We also observed downregulation of BRCA1, accompanied by miR-493-5p upregulation, in artesunate-treated A549/DDP and SBC-3/ADM cells. Ectopic expression of BRCA1, a gene conferring susceptibility to early-onset breast and ovarian cancer ${ }^{[27]}$ and a tumor-suppressive protein ${ }^{[28]}$, was recently reported in patients with metastatic non-small cell lung cancer who received cisplatin-based chemotherapy [19]. Our data also revealed upregulation of BRCA1 mRNA and the BRCA1 protein, as well as an increase in the phosphorylation of PI3K/AKT, a known downstream pathway for BRCA1 ${ }^{[20]}$, in artesunate-treated A549/DDP and SBC-3/ADM cells when miR-493 was silenced. These data pointed to the possible regulatory mechanism of miR-493 in the BRCA1 pathway. Control modes of miRNA for gene expression mainly focus on the directly complementary combination of them to target genes transcript. Data from a bioinformatics analysis suggested that BRCA1 may be a potential target gene for miR-493-5p. The results of the luciferase reporter gene assay confirmed this idea. Based on these data, we conclude that BRCA1/PI3K/AKT signals serve as a downstream pathway for miR-493 and that together they form a molecular mechanism by which artesunate exhibits anti-cell drug resistance activity against lung carcinomas.

In summary, this study is the first to demonstrate the anti-cell drug resistance activity of artesunate against lung carcinoma cells and to shed light on the potential molecular mechanism underlying this activity, which involves miR-493-5p/BRCA1 and the downstream PI3K/AKT pathway. The findings provide evidence for the use of artesunate as a treatment for lung carcinomas.

\section{REFERENCES}

1. Sousa, V., Reis, D., Silva, M., Alarcão, A. M., Ladeirinha, A. F., D’Aguiar, M. J., Ferreira, T., Caramujo-Balseiro, S., and Carvalho, L. (2016) Amplification of FGFR1 gene and expression of FGFR1 protein is found in different histological types of lung carcinoma. Virchows Archiv 469, 1-10

2. Mateen, S., Raina, K., and Agarwal, R. (2013) Chemopreventive and anti-cancer efficacy of silibinin against growth and progression of lung cancer. Nutrition \& Cancer 65 Suppl 1, 3

3. Meshnick, S. R., Taylor, T. E., and Kamchonwongpaisan, S. (1996) Artemisinin and the antimalarial endoperoxides: from herbal remedy to targeted chemotherapy. Microbiological reviews 60, 301-315

4. Pan, H. Z., Lin, F. B., and Zhang, Z. A. (1989) Effect of sodium artesunate on malaria infected human erythrocytes. Proceedings of the Chinese Academy of Medical Sciences and the Peking Union Medical College = Chung-kuo $\mathrm{i}$ hsueh k'o hsueh yuan, Chung-kuo hsieh ho i k'o ta hsueh hsueh pao 4, 181-185

5. Bachmeier, B., Fichtner, I., Killian, P. H., Kronski, E., Pfeffer, U., and Efferth, T. (2011) Development of resistance towards artesunate in MDA-MB-231 human breast cancer cells. PloS one 6, e20550

6. Thanaketpaisarn, O., Waiwut, P., Sakurai, H., and Saiki, I. (2011) Artesunate enhances TRAIL-induced apoptosis in human cervical carcinoma cells through inhibition of the 
NF-kappaB and PI3K/Akt signaling pathways. International journal of oncology 39, 279-285

7. Ma, H., Yao, Q., Zhang, A. M., Lin, S., Wang, X. X., Wu, L., Sun, J. G., and Chen, Z. T. (2011) The effects of artesunate on the expression of EGFR and ABCG2 in A549 human lung cancer cells and a xenograft model. Molecules 16, 10556-10569

8. Nunes, J. J., Pandey, S. K., Yadav, A., Goel, S., and Ateeq, B. (2017) Targeting NF-kappa B Signaling by Artesunate Restores Sensitivity of Castrate-Resistant Prostate Cancer Cells to Antiandrogens. Neoplasia 19, 333-345

9. Roh, J. L., Kim, E. H., Jang, H., and Shin, D. (2017) Nrf2 inhibition reverses the resistance of cisplatin-resistant head and neck cancer cells to artesunate-induced ferroptosis. Redox biology 11, 254-262

10. Papanikolaou, X., Johnson, S., Garg, T., Tian, E., Tytarenko, R., Zhang, Q., Stein, C., Barlogie, B., Epstein, J., and Heuck, C. (2014) Artesunate overcomes drug resistance in multiple myeloma by inducing mitochondrial stress and non-caspase apoptosis. Oncotarget 5, 4118-4128

11. An, X., Sarmiento, C., Tan, T., and Zhu, H. (2017) Regulation of multidrug resistance by microRNAs in anti-cancer therapy. Acta pharmaceutica Sinica. B 7, 38-51

12. Fang, C., Chen, Y. X., Wu, N. Y., Yin, J. Y., Li, X. P., Huang, H. S., Zhang, W., Zhou, H. H., and Liu, Z. Q. (2017) MiR-488 inhibits proliferation and cisplatin sensibility in nonsmall-cell lung cancer (NSCLC) cells by activating the eIF3a-mediated NER signaling pathway. Scientific reports 7, 40384

13. Meng, F., Wang, F., Wang, L., Wong, S. C., Cho, W. C., and Chan, L. W. (2016) MiR-30a-5p Overexpression May Overcome EGFR-Inhibitor Resistance through Regulating PI3K/AKT Signaling Pathway in Non-small Cell Lung Cancer Cell Lines. Frontiers in genetics 7, 197

14. Zhao, L., Feng, X., Song, X., Zhou, H., Zhao, Y., Cheng, L., and Jia, L. (2016) miR-493-5p attenuates the invasiveness and tumorigenicity in human breast cancer by targeting FUT4. Oncology reports 36, 1007-1015

15. Jia, X., Li, N., Peng, C., Deng, Y., Wang, J., Deng, M., Lu, M., Yin, J., Zheng, G., Liu, H., and He, Z. (2016) miR-493 mediated DKK1 down-regulation confers proliferation, invasion and chemo-resistance in gastric cancer cells. Oncotarget 7, 7044-7054

16. Sakai, H., Sato, A., Aihara, Y., Ikarashi, Y., Midorikawa, Y., Kracht, M., Nakagama, H., and Okamoto, K. (2014) MKK7 mediates miR-493-dependent suppression of liver metastasis of colon cancer cells. Cancer science 105, 425430

17. Gu, Y., Cheng, Y., Song, Y., Zhang, Z., Deng, M., Wang, C., Zheng, G., and He, Z. (2014) MicroRNA-493 suppresses tumor growth, invasion and metastasis of lung cancer by regulating E2F1. PloS one 9, e102602

18. Tambe, M., Pruikkonen, S., Maki-Jouppila, J., Chen, P., Elgaaen, B. V., Straume, A. H., Huhtinen, K., Carpen, O., Lonning, P. E., Davidson, B., Hautaniemi, S., and Kallio,
M. J. (2016) Novel Mad2-targeting miR-493-3p controls mitotic fidelity and cancer cells' sensitivity to paclitaxel. Oncotarget 7, 12267-12285

19. Papadaki, C., Sfakianaki, M., Ioannidis, G., Lagoudaki, E., Trypaki, M., Tryfonidis, K., Mavroudis, D., Stathopoulos, E., Georgoulias, V., and Souglakos, J. (2012) ERCC1 and BRAC1 mRNA expression levels in the primary tumor could predict the effectiveness of the second-line cisplatin-based chemotherapy in pretreated patients with metastatic non-small cell lung cancer. Journal of thoracic oncology : official publication of the International Association for the Study of Lung Cancer 7, 663-671

20. Galetti, M., Petronini, P. G., Fumarola, C., Cretella, D., La Monica, S., Bonelli, M., Cavazzoni, A., Saccani, F., Caffarra, C., Andreoli, R., Mutti, A., Tiseo, M., Ardizzoni, A., and Alfieri, R. R. (2015) Effect of ABCG2/BCRP Expression on Efflux and Uptake of Gefitinib in NSCLC Cell Lines. PloS one 10, e0141795

21. Xin, Y., Yin, F., Qi, S., Shen, L., Xu, Y., Luo, L., Lan, L., and Yin, Z. (2013) Parthenolide reverses doxorubicin resistance in human lung carcinoma A549 cells by attenuating NF-kappaB activation and HSP70 up-regulation. Toxicology letters 221, 73-82

22. Efferth, T. (2017) From ancient herb to modern drug: Artemisia annua and artemisinin for cancer therapy. Seminars in cancer biology

23. Chen, Y., Gao, Y., Zhang, K., Li, C., Pan, Y., Chen, J., Wang, R., and Chen, L. (2015) MicroRNAs as Regulators of Cisplatin Resistance in Lung Cancer. Cellular physiology and biochemistry : international journal of experimental cellular physiology, biochemistry, and pharmacology 37 , $1869-1880$

24. Lehmann, U., Streichert, T., Otto, B., Albat, C., Hasemeier, B., Christgen, H., Schipper, E., Hille, U., Kreipe, H. H., and Langer, F. (2010) Identification of differentially expressed microRNAs in human male breast cancer. BMC cancer 10, 109

25. Ueno, K., Hirata, H., Majid, S., Yamamura, S., Shahryari, V., Tabatabai, Z. L., Hinoda, Y., and Dahiya, R. (2012) Tumor suppressor microRNA-493 decreases cell motility and migration ability in human bladder cancer cells by downregulating RhoC and FZD4. Molecular cancer therapeutics 11, 244-253

26. Zhou, W., Zhang, C., Jiang, H., Zhang, Z., Xie, L., and He, X. (2015) MiR-493 suppresses the proliferation and invasion of gastric cancer cells by targeting RhoC. Iranian journal of basic medical sciences 18, 1027-1033

27. Abel, K. J., Xu, J., Yin, G. Y., Lyons, R. H., Meisler, M. H., and Weber, B. L. (1995) Mouse Brca1: localization sequence analysis and identification of evolutionarily conserved domains. Human molecular genetics 4, 2265-2273

28. Turner, J. G., Dawson, J., and Sullivan, D. M. (2012) Nuclear export of proteins and drug resistance in cancer. Biochemical pharmacology 83, 1021-1032Figures legends 